\title{
ADVANCED SIMULATION CAPABILITY FOR ENVIRONMENTAL MANAGEMENT (ASCEM): AN OVERVIEW OF INITIAL RESULTS
}

\author{
Mark Williamson, ${ }^{*}$ Juan Meza, $†$ David Moulton, $\ddagger$ Ian Gorton,§ Mark Freshley, $\S$ \\ Paul Dixon, $\ddagger$ Roger Seitz, $\llbracket$ Carl Steefel, $\dagger$ Stefan Finsterle, $\dagger$ Susan Hubbard, $\dagger$ \\ Ming Zhu, ${ }^{*}$ Kurt Gerdes, ${ }^{*}$ Russ Patterson,\# and Yvette T. Collazo* \\ *U.S. Department of Energy, Office of Environmental Management, Washington, DC, USA \\ $\nmid$ Lawrence Berkeley National Laboratory, Berkeley, CA, USA \\ $\ddagger$ Los Alamos National Laboratory, Los Alamos, NM, USA \\ §Pacific Northwest National Laboratory, Richland, WA, USA \\ IISavannah River National Laboratory, Aiken, SC, USA \\ \#U.S. Department of Energy, Carlsbad, NM, USA
}

\begin{abstract}
The US Department Energy (DOE) Office of Environmental Management (EM) determined that uniform application of advanced modeling in the subsurface could potentially help reduce the cost and risk associated with its environmental cleanup mission. In response to this determination, the EM Office of Technology Innovation and Development (OTID), Groundwater and Soil Remediation $(G W \& S)$ began the program Advanced Simulation Capability for Environmental Management (ASCEM). ASCEM is a state-of-the-art scientific tool and approach for integrating data and scientific understanding to enable prediction of contaminant fate and transport in natural and engineered systems. This initiative supports the reduction of uncertainties and risks associated with EM's environmental cleanup and closure programs by better understanding and quantifying the subsurface flow and contaminant transport behavior in complex geological systems. This includes the long-term performance of engineered components, including cementitious materials in nuclear waste disposal facilities that may be sources for future contamination of the subsurface. This article describes the ASCEM tools, approach, and reports and ASCEM programmatic accomplishments completed in 2010.
\end{abstract}

Key words: Environmental management; Simulation; Model; Groundwater; ASCEM

\section{BACKGROUND: INTRODUCTION TO EM NEEDS}

Fifty years of nuclear weapons production and government-sponsored nuclear energy research in the US during the Cold War generated large amounts of radioactive wastes, spent fuel, excess plutonium and uranium, thousands of contaminated facilities, and contaminated groundwater and soil. During most of that half century, the nation did not have the environmental regulatory structure or nuclear waste remediation technologies that exist to- day. The result was a legacy of nuclear waste that was stored and disposed of in ways now considered unacceptable (11).

At the end of US Government Fiscal Year 2010 (FY10), EM had 18 funded sites. Estimates report these sites to contain 40 million $\mathrm{m}^{3}$ of contaminated soil and 6.4 trillion $\mathrm{L}$ of contaminated groundwater (7). Current groundwater and soil remediation challenges that will continue to be addressed in the next decade include cost-effective characterization, remediation, and monitoring of contaminants in the vadose zone and groundwater. 
Many of the contaminated sites require additional characterization, most require final remediation decisions, and all of them will require long-term monitoring.

Although EM has made significant progress in its restoration efforts at sites such as Fernald and Rocky Flats, many of the remaining challenges are the most complex ever encountered, especially at larger sites such as Savannah River, Oak Ridge, and Hanford. These sites contain some of the largest groundwater and soil contamination problems and subsequent remediation responsibilities in the world in terms of the sheer volume of affected groundwater and soil, number of plumes, complexity of hydrogeologic settings, and diversity of contaminant types. In response to a congressional request, DOE identified key engineering and technology gaps organized into a research and development (R\&D) roadmap to support EM cleanup efforts (4). In a review of the roadmap, the National Research Council (NRC) of the National Academies provided advice to EM for addressing principal science and technology gaps (6). Table 1 shows the principal technology gaps identified by DOE in their groundwater and soil remediation program and NRC's ranking of their R\&D priority.

To address these gaps, NRC provided a series of recommendations, one of which focused on the development and use of advanced computational models. NRC advised that these modeling tools should:

1. Incorporate understanding of site geohydrology and contaminant geochemistry with the goal of improving currently insufficient scientific knowledge base (GS-1).

2. Include robust models of caps/covers, barriers, and cementitious materials/waste forms (GS-3).
3. Incorporate appropriate uncertainty (GS-1, GS2, GS-3, GS-4).

4. Account for natural and anthropogenic spatial and temporal changes together with field data to calibrate these models (GS-3, GS-4).

5. Develop predictive capabilities to understand contaminant behavior and to support developing and implementing effective and sustainable remediation approaches (needs previously identified in internal workshops and reviews $(4,5)$.

In response to the National Academy of Science (NAS) and internal DOE review recommendations, and to address key challenge areas including GS-1, GS-3, and GS-4, EM has launched the Advanced Simulation Capability for Environmental Management (ASCEM) program to develop the tools and approaches necessary to use modeling capabilities to predict future contamination distributions within the subsurface based on historical data and possible remedial actions.

\section{INTRODUCTION TO ASCEM}

ASCEM is described as a tool and approach because it is recognized that no single tool will completely represent the risk and/or performance assessment of a site. The collection of tools and actions required to complete the risk and/or performance assessments can, however, be incorporated into an effective and efficient standardized approach. The success of the ASCEM program will be measured by the acceptance of the tools and approaches by end users. This acceptance will almost certainly require that ASCEM be easy to use, accurate, and able to meet user requirements.

ASCEM is being created through collaboration between eight DOE National Laboratories and the

Table 1. Principal Science and Technology Gaps in the EM Cleanup Technology Roadmap and Their R\&D Priorities as Stated by the National Research Council (4)

\begin{tabular}{|c|c|c|}
\hline GS\# & Gap & Priority \\
\hline GS-1 & Contaminant behavior in the subsurface is poorly understood. & high \\
\hline GS-2 & $\begin{array}{l}\text { Site and contaminant source characteristics may limit the usefulness of baseline subsurface remediation } \\
\text { technologies. }\end{array}$ & medium \\
\hline GS-3 & Long-term performance of trench caps, liners, and reactive barriers cannot be assessed with current knowledge. & medium \\
\hline GS-4 & Long-term ability of cementitious materials to isolate wastes is not demonstrated. & high \\
\hline
\end{tabular}


Cementitious Barriers Partnership (CBP). The team is primarily composed of members from Los Alamos National Laboratory (LANL), Lawrence Berkeley National Laboratory (LBNL), Pacific Northwest National Laboratory (PNNL), Oak Ridge and Savannah River National Laboratory (SRNL), Idaho National Laboratory (INL), Lawrence Livermore National Laboratory (LLNL), and Argonne National Laboratories (ANL). The CBP is a collaborative program sponsored by the EM Office of Waste Processing (3). The objective of the CBP is to develop a set of computational tools to improve understanding and prediction of the longterm structural, hydraulic, and chemical performance of cementitious barriers and waste forms used primarily in disposal. The work of the CBP will be incorporated in the ASCEM codes.

The ASCEM program is organized into three technical thrust areas: Site Applications, Platform and Integrated Toolsets to provide the user interfaces (referred to as Platform from here onward), and the Multiprocess High Performance Computing (HPC) Simulator, which constitutes the computational engine (Fig. 1). An overview of the thrust areas is provided below.

\section{Site Applications}

As previously stated, success of the ASCEM program requires end user acceptance. The Site Applications Thrust area provides the main link between ASCEM and the EM community's modeling and regulatory needs. To achieve this acceptance the ASCEM team has engaged the user community in the early stages of the ASCEM development. The Site Applications Thrust area incorporates a "user interface" task focused on establishing contact with end users, soliciting their input about ASCEM development plans, and conveying the feedback to members of the HPC and Platform Thrust areas responsible for the tool and code development.

End user involvement in ASCEM includes performance assessment (PA) and risk assessment practitioners, decision makers, oversight personnel, and regulators who are engaged in the DOE cleanup mission. Solicitation of end user feedback was critical during initial ASCEM development to ensure that user needs are incorporated into the framework. Subsequent and consistent engagement is key to developing user acceptance and eventual application of the ASCEM toolsets at DOE sites.

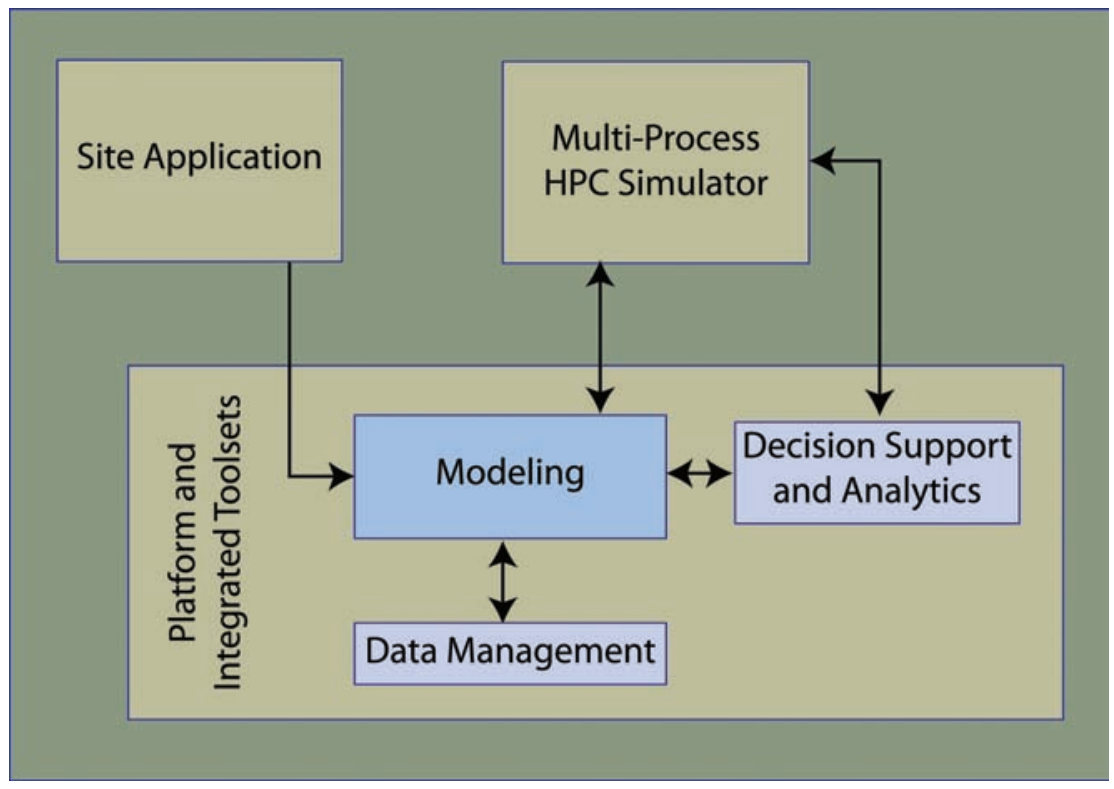

Figure 1. The three technical thrust areas of ASCEM: Multiprocess HPC Simulator, Platform and Integrated Toolset, and Site Applications. 
Over the long term, the Site Applications Thrust includes several tasks designed to engage and support end users, including site demonstrations, development of protocols, documentation and work flows, and training and support in the use of ASCEM tools.

Prior to developing code for ASCEM, the site applications group obtained input from potential end users. This information was gathered through various mechanisms described below and was summarized in User Suggestions and State of Practice for Development of ASCEM Requirements (12), which provided feedback for development requirements for the HPC and Platform Thrust areas. It also provided suggestions for potential test cases and demonstrations for use in future Site Applications activities.

Input was obtained through different activities including:

1. Interviews with potential users at DOE sites: Eighteen separate interviews were conducted with National Laboratory, site contractor, and/ or DOE staff at Los Alamos, Idaho, Savannah River, Lawrence Berkeley, Pacific Northwest, Lawrence Livermore, Oak Ridge National Laboratories, Hanford, and Portsmouth/Paducah sites. The interviews ranged from detailed oneon-one or small group discussions to larger discussions involving a mixture of DOE, contractor, and National Laboratory personnel. Each interview took 1-2 $\mathrm{h}$ and involved open discussions of ASCEM and end user expectations. Generally, site personnel reacted positively in the interview process and agreed that the interviews facilitated greater understanding of ASCEM goals.

2. Discussions with DOE Office of Science Subsurface Biogeochemical Research (SBR) program participants: SBR conducted its principal investigator meeting in March of 2010. The meeting included a breakout session on "Modeling and Simulation of Subsurface Systems," which was designed to address a series of issues including:

A. Identifying the weakest links in reactive transport models in terms of processes or parameters limiting their predictive ability.
B. Discussing the modeling role in experimental design and interpretation.

C. Identifying new theories for HPC model development and application.

D. Discussing the importance of computer science and applied mathematics for model improvements.

E. Incorporating new kinds of characterization and other data into models to improve predictive abilities.

F. Optimizing the relationship between required data and increasing levels of complexity in recognition that future models will be data limited.

G. Attendance at this meeting and subsequent discussions with the participants provided the ASCEM team with input from the modelers currently working within the EM community.

3. Participation in a Performance Assessment Community of Practice (PA CoP) technical exchange: Through this technical exchange, a broad cross-section of potential users provided feedback on ASCEM. The PA CoP technical exchange provided a venue for performance and risk assessment practitioners, decision makers, and regulators to share experiences with both ASCEM and CBP developers.

4. Reviews of Current Performance Assessment Practices: The ASCEM team reviewed documentation from recent performance assessments conducted at DOE sites to develop perspectives regarding current practices. This process included a review of codes and data used in recent performance assessments and composite analyses prepared in accordance with DOE requirements (5). In general, performance assessment analyses support radioactive waste disposal actions, while risk assessments are conducted to support remediation decisions (although there can be some overlap depending on the regulations governing a given disposal facility). The review was designed to identify the codes, methodologies, main assumptions, and key data sets used in these analyses.

During the course of these discussions, the following common suggestions arose:

1. Current regulatory modeling approaches have 
been adequate, but improved capabilities are needed to address future challenges.

2. Modeling needs to be integrated with field sampling, demonstrations and monitoring for a holistic approach to decision making.

3. Structured documentation, transparency of assumptions, and ease of use are important for gaining broad acceptance.

4. A graded and iterative approach is critical, including a continuum of modeling complexity from screening to detailed process models.

5. Improved capabilities for source-term models (i.e., barrier and waste form degradation and reactive transport for release processes) will help with key challenges.

6. HPC needs to be leveraged for better efficiency of uncertainty analyses and process modeling for complex systems.

7. End users and decision makers need to be involved in the development process, especially in the Platform Thrust and demonstrations and testing.

8. Processes related to surface exposure pathways need to be addressed (i.e., intrusion scenarios and biotic transport).

Actively seeking end user input has proven invaluable in introducing ASCEM to the user community and helping the ASCEM team to understand user needs. In addition, this outreach also provided a number of general suggestions, as well as suggestions for a number of potential test or demonstration problems that will be considered during selection of future demonstration sites for the Site Applications Thrust area.

\section{Platform and Integrated Toolsets}

In the Platform and Integrated Toolsets Thrust area, ASCEM is creating a comprehensive collection of user-facing tools. The Platform will enable site application users to harness HPC simulation capabilities for environmental management tasks and support the related model development and analysis functions.

The Platform incorporates a set of tools into a consistent user interface that permits a modeling workflow that is flexible, maintains quality assurance procedures and data integrity, and supports user efficiency.
The components of the Platform Thrust include a number of toolsets for: 1) Model setup and analysis; 2) Parameter estimation; 3) Uncertainty quantification; 4) Risk assessment; 5) Decision support; 6) Information and data management; 7) Visualization.

All of the tools within the Platform require access to data organized within the ASCEM management system. Interaction with the HPC simulator is coordinated by this core platform. ASCEM, including the Platform, is being developed as an opensource technology executable on standard client and server platform operating systems such as Linux and Windows. The design and development actively avoids dependencies on specific operating systems and runtime environments to ensure broad portability.

The Platform and Integrated Toolset Thrust will provide a standardized user interface, enabling end users to create inputs, analyze outputs, and manage data associated with running simulations and performance and risk assessments. Under this thrust area, ASCEM is using a modular (or "interoperable") approach to code development, facilitating iterative and graded modeling systems that allow end user customization for specific applications without the need for specialized computational or code development expertise. This is accomplished by defining rigorous programming "interfaces" for each module (where an interface defines access to a module while hiding the details of its implementation). By using a common base platform available to all, this interoperable approach will support cooperation among numerous modeling groups with different methodologies and applications. This methodology has been successful in the past and is broadly used in similar advanced software engineering approaches, for example, within the DOE Office of Science, Advanced Scientific Computing Research (ASCR) Office's Scientific Discovery through Advanced Computing (SciDAC) program, and the DOE National Nuclear Security Administration (NNSA), Advanced Simulation and Computing (ASC) program. This modular approach will also be used to develop process models that are imperative for successfully implementing performance and risk assessment approaches.

A key part of the Platform is the user interface. The ASCEM user interface is the means by which 
users interact with ASCEM applications, within the larger context of interacting with other non-ASCEM applications, scientists, and the physical world. The objective of user interface design for the ASCEM project is to provide all scientists and system users' seamless access to the Platform and external tools, including the data and models needed for the execution of simulations, and the associated visualization and analysis tools.

Usability engineering principles and practices such as detailed task analysis, competitive analysis, participatory and iterative user-centered design, mental models, user personas, low-fidelity prototypes, interactive prototypes, and usability testing have repeatedly demonstrated their effectiveness in reducing software development and deployment costs and improving end users' efficiency, situational awareness, and productivity. These techniques and processes have been effectively applied in large software projects over a multitude of domains, including both commercial and scientific systems, and are being followed in the development of ASCEM.

As used in ASCEM, a user-centered design takes into account users cognitive abilities, organizational constraints, and customs and precedent. Further, end users are involved throughout the project. Integrating usability design throughout the entire development and deployment process has been shown to yield more effective end results. The standard approach to user-centered design includes, at a minimum, a needs assessment and scenario and task creation, followed by an initial design with a focus on user involvement and scaffolding. Rapid prototyping and heuristic evaluation will be included in the design process to reduce the overall cost of the user interface (UI). The ASCEM team will use this methodology to build a usable and effective interface that promotes user efficiency and accuracy during all aspects of the ASCEM modeling process.

Software Interfaces. The ASCEM Platform will leverage a number of well-established opensource toolsets in its design and construction. The design will ensure that the toolsets and components built on top of them are loosely coupled by minimizing software interfaces to promote a flexible, modifiable software system.

Figure 2 shows a simple depiction of the inter- faces for the various components of the Platform. The Core Platform and Data Management components will provide a programmatic interface for all the toolsets to access the data required for a modeling project.

In addition to internal interfaces, the following interfaces will also be supported:

1. Interfaces to queue systems that control access to large-scale computational resources.

2. Interfaces to commercial and open-source tools used in the modeling and analysis process. These interfaces will be achieved through data integration. This will require the Platform to supply import/export functions for each supported tool. Examples of possible supported tools are GMS, Resrad, and EarthVision. The exact list of tools that are integrated, and their priorities, will be dictated by the requirements, with input from user interface activities conducted by the ASCEM Site Application Thrust.

3. The ability to use existing flow and transport simulators, if identified as a requirement in the Site Applications Thrust, will be supported by the Platform component for launching external simulators. This component will assume all the necessary input files are supplied.

4. Interface protocols to allow access to remote computational file transfer platforms, such as ssh and sftp.

5. Web-based HTTP protocols for various component integration requirements.

The Platform is organized as a set of integrated software components as depicted in Figure 2. A brief outline of the expected functionality of each is provided below.

Core Platform. The Core Platform (CORE) provides user access to ASCEM functions and data. The software developed for this capability will include an integrated Graphical User Interface (GUI), tools for automated job launching and monitoring, and a set of Application Programmer Interfaces (APIs), which provide the basic framework for constructing and integrating ASCEM Toolsets.

CORE will provide infrastructure to enable routine tasks such as data sharing to be automated and to facilitate collaboration among project partici- 


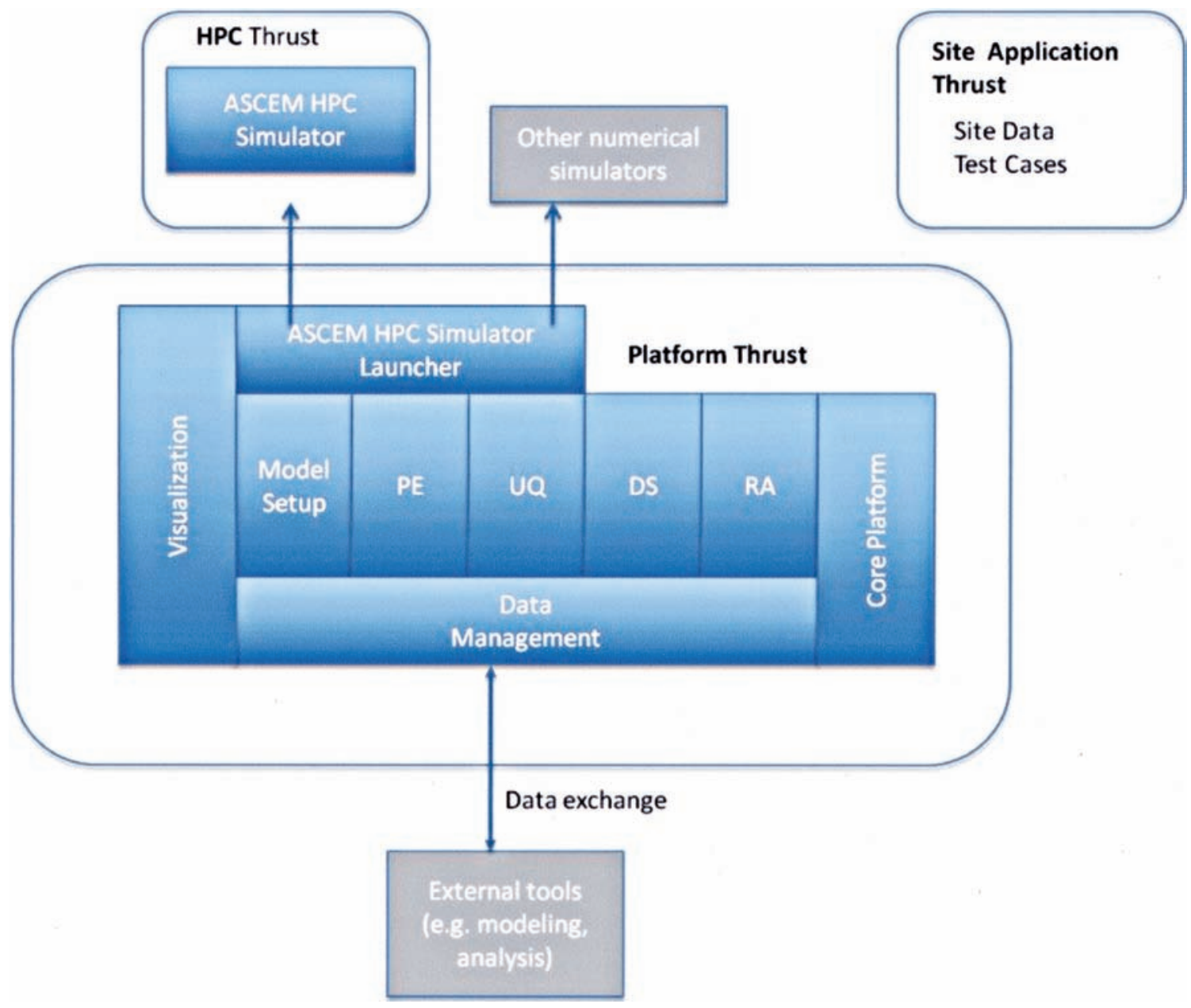

Figure 2. ASCEM Platform Interfaces. Data Management is required to interact with each of the toolsets including Visualization, Model Setup, Parameter Estimation (PE), Uncertainty Quantification (UQ), Decision Support (DS), Risk Analysis (RA), and the Core Platform.

pants. CORE will specify the primary integration points for the Platform, including access to underlying data management and simulation infrastructures. The overall goal is to provide both command line tools and a GUI to support a flexible, streamlined, and efficient modeling process.

There are many types of tools that will be used with the ASCEM platform, and the level of integration will vary. Tools are grouped into categories based on both the type of tool and the expected level of integration.

ASCEM Tools. This category covers tools that are developed by the ASCEM team. Some capabilities, such as visualization, will likely be built upon existing tools, and where the ASCEM team has source code access and expertise with the development of these tools, will be integrated at the appropriate level. All of these tools will be available in the Platform and the various toolsets it supports. The functions provided by many of these tools will also be available at the command line. Examples of these include the tools associated with the other Platform Toolsets as well as search functionality, browsing capability, and model comparison tools.

Integrated Tools This category of tools includes third-party open-source tools that can provide targeted capabilities through leveraging. These tools can generally be scripted at the command line and are used to create data views. Integration with ASCEM will require developing 
scripts that result in new or transformed outputs. Source code for these tools will not normally be modified by the ASCEM team. Examples of these tools may include Gnuplot (and similar generalpurpose plotting tools), seismic data processing software, R statistical scripting language, and file transfer tools.

External Tools. These are third-party tools that will be run outside the ASCEM platform, and may be integrated through appropriate data input/output exchange mechanisms. Various considerations inhibit tighter integration, including licensing or policy restrictions, costs, and closed, monolithic UI environments. These tools can be further broken down into categories by function including modeling tools, assessment models, alternative flow and transport models, and analysis tools. This functionality will be particularly important for validating the ASCEM models.

From the CORE perspective, the job execution framework will support any of the flow and transport models, as they will be treated as black boxes with inputs and outputs. Integration of other modeling or analysis tools will be at a very high level only via data exchange into and out of the platform. The Model Setup Toolset will be responsible for creating data translation tools that enable seamless use of selected tools. CORE will integrate these tools to provide on-demand data conversion.

Model Setup and Analysis Toolset. Creating the Model Setup Toolset consists of two primary tasks. The first is to provide tools that allow the ASCEM end user to create the input data structures necessary to define the system geometry, hydrogeological and biogeochemical processes and constitutive parameters, initial and boundary conditions, source-sink terms, solver control parameters, and other information necessary to run a subsurface flow and reactive transport model calculation and to control the parameter estimation and uncertainty quantification algorithms. The second is to develop interfaces to the computational models that translate the model setup input files and data structures into the parameters, vectors, and arrays that the numerical analysis tools and solvers need to run computations.

This toolset will provide the capability for generation of simulator input files, including initial and boundary conditions, sources and sinks, and material properties. It will link the geologic framework model to grid-generation algorithms, and provide mapping or interpolation of material properties and parameters between different grids. Observational data will also be linked to facilitate history matching and inverse modeling. This toolset will generate model attribute summary reports to facilitate model-data and model-model comparisons.

Parameter Estimation Toolset. Parameter Estimation (PE) is a key part of the model development process. It relates a conceptual model describing the conditions at the site to actual field data. At the same time, it allows the determination of model-related, process-dependent, and sitespecific parameter values that cannot be reliably obtained from independent sources. Moreover, it examines the ability of the model to represent the system of interest, points towards aspects in the model that need to be refined, and helps quantify and potentially reduce the uncertainty of subsequent model predictions.

The PE Toolset provides the methodology and tools needed to estimate values for selected model parameters. Two classes of approaches are considered: 1) Model-based data inversion using the multiprocess simulator(s) supported by ASCEM as the computation tool, and 2) Analysis approaches that combine multiple sources of information for Bayesian parameter estimation without invoking the multiprocess simulator.

In the first approach, values of model parameters-which may, for example, represent material properties, coefficients in process descriptions, initial and boundary conditions, geometric features, and other input parameters of the multiprocess simulator-are inferred from measured data. This is done by evaluating or minimizing some function of the difference between these measured data and the corresponding simulated system response at discrete points in space and time. This parameter estimation approach is also described as automatic model calibration or history matching and may be performed within an optimization or Bayesian sampling framework. In addition to estimating parameter values, the PE Toolset will calculate various measures that evaluate both the quality of the match and that of the estimates. As a byproduct, sensitivity coefficients, information content of data 
sets, parameter identifiability, and other diagnostic measures and analyses will be provided.

In the second approach, property values are estimated by combining information from multiple sources through conditioning (i.e., geostatistical and Bayesian methods). Data may include geological, hydrological, hydrogeological, geochemical, and geophysical field and laboratory measurements. They may be quantitative or qualitative. Relationships between parameters under estimation and available data are not provided deterministically, but through probabilistic models. Property fields generated by this approach may be directly assigned to the model for subsequent flow and reactive transport simulations, or they may be used as prior information (or starting model) for further inversion of other types of data.

In summary, the ASCEM Parameter Estimation Toolset provides inverse modeling capabilities for the estimation of input parameters of the multiprocess simulator based on measured data, performs residual and parameter uncertainty analyses, estimates properties through conditioning of data of different types without invoking the multiprocess simulator, and provides optimization algorithms for use by other toolsets.

Uncertainty Quantification Toolset. Uncertainty quantification (UQ) entails a number of services that involve propagation of input variations and uncertainties through a forward model. The basic processes include:

1. Generating ensembles of parameter and conceptual model variations;

2. Forward error/uncertainty propagating;

3. Summarizing model output produced from an ensemble of forward model runs;

4. Analyzing model outputs to: A) Determine key sources of uncertainty, and B) Develop strategies for efficiently reducing uncertainty;

5. Sensitivity analysis.

The high computational demands of the forward model make it necessary to use methodologies that make the most effective use of the limited number of forward model runs that can be carried out. These methods include: 1) Forward model response surface approximations; and 2) Generation of input parameter sets for relatively small ensembles of forward model runs that will approximate the performance of large ensembles.

Using these techniques, the UQ Toolset will optimize the design of experiments to reduce the required number of simulations necessary to achieve the desired results.

Risk Assessment Toolset. The Risk Analysis (RA) Toolset provides a comprehensive risk resource with the flexibility and adaptability to support all regulatory environments (or other basis for decisions) as well as synthesizing risk with other primary data/information components in support of data collection and decision processes. This approach, while including the assessment of risk to aid in protecting human health and the environment, also takes into account the broader decision context. Of primary importance is the identification of the decision objectives and decision drivers to ensure that data needed for and from the risk assessment are provided as well as determining the level of effort and detail that is required for the risk evaluation.

Some risk factors include site complexity (number of chemicals, spatial and temporal considerations, and exposure pathways), availability of toxicity data and Applicable or Relevant and Appropriate Requirements (ARARs), quality of characterization data, anticipated remedial actions needs for fate and transport modeling, economic impacts, and probability of technology failure. These considerations are also determining factors in the complexity of fate and transport modeling required.

The RA Toolset will provide data, tools, and guidance to enhance the integration of risk in the EM decision process. The toolset will include a repository for DOE and regulatory guidance, standard risk parameters, data, and risk tools. It will also provide user support to facilitate analyses of impacts from current and future exposure to contaminants of concern to human health and ecological endpoints, as well as economic and technological considerations.

The requirements of the RA Toolset are based on the primary steps followed in the risk analysis process:

1. Data Compilation and Evaluation: Provides tools and information to facilitate data collec- 
tion and assessment in support of decision drivers and data quality objectives.

2. Exposure Assessment: Provides tools to aid the user in the evaluation of site characteristics and contaminant data as a basis for the development of exposure assumptions.

3. Toxicity Assessment: Provides tools and data to aid the user in the presentation of potential adverse health effects associated with chemicals/ radionuclides of potential concern.

4. Risk Characterization: Provides models for the calculation of risk or dose that will allow the user to define the organization of output based on land use scenarios, exposure pathway, media, exposure route, and chemicals/radionuclides.

This process will be incorporated into the ASCEM RA Toolset to provide end users with the best available data for decision support.

Decision Support Toolset. The Decision Support (DS) Toolset will provide ASCEM users (modelers, managers, regulators, stakeholders) with a computational framework for decision making. A major goal of the DS Toolset is to estimate the impact of conceptual model elements, model parameters, and model predictions (and their respective uncertainties) on the decision-making process. The DS Toolset also addresses the iterative nature of environmental management investigations and decision making, which includes adjustment of decision objectives and environmental management goals.

The analyses of modeling results for DS will require the execution of a series of different model runs. These include:

1. Forward Simulations: A series of independent forward model runs.

2. Sensitivity Analyses: Exploration of sensitivity of model predictions to selected model parameters and conceptual model elements.

3. Uncertainty Analyses: Quantification of uncertainty in model parameters, model predictions, and conceptual model elements.

4. Inverse Analyses (calibrations): Identification of plausible parameter space based on observation data or other target values.

5. Risk Evaluations: Evaluation of repercussions of decisions due to uncertainties in model parameters, model predictions, and conceptual model elements.

The DS Toolset will facilitate analyses using the functionality provided by the other Platform Toolsets. In this way, the DS Toolset will provide decision-making context to tasks performed by other Toolsets, such as Model Setup (MS), Parameter Estimation (PE), Uncertainty Quantification (UQ), and Risk Analysis (RA). The guidance from the DS Toolset to the other Platform Toolsets will be related to various aspects of model development, including model setup, model parameters, observation data (calibration targets), model predictions, and control parameters (e.g., resolution, precision, accuracy, and confidence levels).

The goal for the DS Toolset is to define model development based on the decision-making needs. In many cases, decision making about environmental management may not need any model development. The decision makers will be integrally involved throughout the entire process of investigation, remediation, and long-term monitoring of a site to ensure their decision-making objectives are well represented. This will be strongly facilitated by ASCEM capabilities. The decision-making process is expected to be iterative and the objectives of the decision makers, with consideration of acceptable and unacceptable uncertainties, are expected to change over time as additional data are obtained.

Data Management Toolset. The Data Management Toolset will consist of a data and information management infrastructure that is accessible to all the toolsets of the ASCEM Platform. The data stored in the data management infrastructure will include the set of data used to develop a site characterization, parameter databases, conceptual and numerical models, and inputs and outputs from simulations executed by the ASCEM HPC simulator and the various toolsets. These collections of different data types will have to be stored in a common database. Data need to be organized, versioned, easily accessible, and effectively managed to ensure consistency, reproducibility, and traceability of ASCEM analyses.

At most environmental management sites these 
data are typically organized into separate databases such as a well database, a tank database, and a contaminants database. In addition, many datasets are available as files on individual users systems or on shared disk systems. However, in the ASCEM data management system, the ability to identify collections of these data is important. To incorporate these collections, the ASCEM data management system will organize these disparate collections into a single database that will contain data entries provided by end user activities. Each data object stored in the ASCEM system will have additional information associated with it, describing its type, place of measurement, site designation, database that is the source of the data, as well as format and context-dependent information that is identified as important for searching.

The ASCEM data management system will enable sharing of data sets, association of analyses with simulation results, running in a flexible but unambiguous way, automation of management of the large volumes of data associated with sensitivity and validation studies, and maintenance and viewing of provenance between the various data items and simulation runs. It will also be possible to use data extracted from established data management systems at user sites, or to have information in the ASCEM database on how to get such data.

Datasets produced by simulations are spatiotemporal in nature. For the purpose of analysis and visualization, it is often necessary to select subsets of data not only based on space and time, but also by the values of a variable (such as values that identify a plume, the types of contaminant, regions with high levels of contamination, and types of remediation activities). This requires efficient indexing of entire datasets to allow fast exploration and interactive visualization. Such indexing techniques will be an integral part of the data management system.

In the ASCEM program, a holistic approach is being taken, and all model input, output, and control files will be managed collectively, versioned, and supported by data access tools that make the modeling task more effective and efficient. The ASCEM data management toolset will support the concept of a "collection of data objects" to support such groupings, as well as metadata at both the file and data collection level. Initially, the data and information management will be centralized. Based on future needs, the data may be partitioned and shared in a distributed fashion.

Visualization Toolset. The Visualization Toolset plays an important role in the ASCEM Platform as a means of viewing different aspects of the conceptual model (i.e., parameterization of the site model and uncertainty quantification analysis) and viewing and analyzing simulation output and derived quantities (i.e., volume of contaminant). The output of the visualization toolset will consist primarily of images (2D and 3D) and movies. In addition, the visualization/analysis activity will enable researchers and site managers to interact with site conceptual models and simulation, output the data from different perspectives, isolate regions of interest, and perform analyses of the simulation output.

Visualization of diverse types of data represents a considerable challenge with respect to meeting users needs. Different formats are used to represent site conceptual models, and a variety of commercial visualization tools are used. The Visualization Toolset will address the issue of multiple heterogeneous data formats. As ASCEM development proceeds, there will be more opportunities to collect and categorize data formats and assess which specific visualization/display tools are appropriate to use for a given type of data.

In order to accommodate these different types of data, two ways to incorporate visualization tools in the ASCEM Platform are being pursued:

1. Standalone: In this approach, the Core Platform launches the Visualization Toolset with a set of parameters (i.e., data file names), and the visualization application runs as an executable standalone. The end user interacts directly with the visualization application's graphical user interface.

2. Embedded: In this case, the Core Platform launches a visualization application with a complete set of parameters that would include name of input data file, visualization algorithms to execute, and settings for the algorithms. The Core Platform will then "harvest" image(s) from the visualization application and display them to the user.

As visualization tools are developed for the site conceptual model, simulation output, and data output from various platform tasks, ASCEM develop- 
ers will evaluate which approach is most appropriate to meet specific user requirements. It is expected that visualization will be required to support 3D visualizations of the site conceptual model that a user can interact with, displaying and examining the output of simulation runs performed by the ASCEM multiprocess HPC simulator, calculation of quantities of interest based on gridded visualization data sets, and to support other tasks in the Platform Thrust (i.e., parameter estimation, risk assessment, uncertainty quantification, and model setup) with visualization and analysis tools.

\section{Multiprocess High Performance Computing Simulator}

The third thrust area, the Multiprocess HPC Simulator, will provide the simulation capabilities necessary for the modeling of complex EM sites. The HPC Simulator will provide a flexible and extensible computational engine to simulate the coupled processes and flow scenarios described by the conceptual models developed with the ASCEM Platform using a graded approach. The graded and iterative approach to assessments naturally generates a suite of conceptual models that span a range of process complexity, potentially coupling hydrological, biogeochemical, geomechanical, and thermal processes. To enable increasing complexity of environmental management simulations to support remediation and site closure issues, ASCEM is taking advantage of emerging petascale computers that handle hundreds of thousands of simultaneous process streams of information. Their use will facilitate improved uncertainty quantification and, when necessary, the use of more complex models in lieu of simplifying assumptions. These HPCcapable tools will be available on platforms from clusters to desktop computers. While there is a clear recognition that many problems will not require high-end computing capabilities, there is a clear need for additional computing resources. In the rapidly changing world of computing, desktop computing capabilities such as multicore computer architectures available today were only available on large-scale computers a few years ago. This quick advancement is expected to continue into the future and the ASCEM approach is designed for these advancements.

The ASCEM Multiprocess HPC Simulator has been named Amanzi-the Zulu word for water. Amanzi is a flexible and extensible computational engine that will simulate the coupled processes described by the conceptual models developed using the ASCEM Platform. These conceptual models span a range of process complexity in flow and reactive transport. Detailed mathematical descriptions of these process models are provided in (13) and summarized below.

A rock, sediment, or soil mass consisting of aggregates of mineral grains and pore spaces or voids is referred to as a porous medium. An actual porous medium is a highly heterogeneous structure containing physical discontinuities marked by the boundaries of pore walls, which separate the solid framework from the void space. Although it is, at least in principle, possible to describe this system at the microscale of individual pores, such a description rapidly becomes a daunting and currently impossible task as the size of the system increases and many pore volumes become involved. It is therefore necessary to approximate the system by creating a more manageable one. One quantitative description of the transport of fluids and their interaction with rocks is based on a mathematical idealization of the real physical system referred to as a continuum. In this approach, the discrete physical system, consisting of aggregates of mineral grains, interstitial pore spaces, and fractures, is replaced by a continuum representation in which physical variables describing the system vary continuously in space. Allowance is made for the possibility of a discrete set of surfaces across which discontinuous changes in physical properties may occur. In the continuum representation of the real physical system, solids and fluids coexist simultaneously at each point in space. This approach is implemented in Amanzi.

There are many formulations of the process models for contaminant transport that one could present. ASCEM starts with conservation of mass, momentum, and energy in the presence of chemical reactions for a multiphase/multicomponent system. This leads to basic flow equations describing nonisothermal, multiphase, multicomponent flows in heterogeneous porous media. Typically, a general formulation of this type leads to more unknown variables than the number of equations (which does not allow for a unique solution to the problem). 
Therefore, additional information is needed to completely describe the problem. One simple possibility to accomplish this is by stating the volumetric water content as a function of the water potential or vice versa.

Flow. Using the continuum approach and Darcy's Law, Richards' Equation is implemented by Amanzi to represent saturated and unsaturated flow (subsequent versions of Amanzi will include multiphase flow). Darcy's Law gives a relationship between velocity and pressure or discharge and pressure gradient across a system. It essentially replaces the momentum equation. There has been much research to support the validity of Darcy's Law (10). Most references give the applicability of Darcy's Law to be for laminar flows with Reynolds numbers less than 10 using the pore throat diameter for soil as the reference length.

It is also assumed that thermodynamic equilibrium (mechanical and thermal) exists for each grid block. Subgrid-scale features will often play a prominent role in multifluid simulations. Faults and fractures will likely be fast paths for contaminant transport and can be treated in some cases as equivalent porous media, or, alternatively, with multiple porosity models. Similarly, rate-limited diffusion from clay inclusions can be modeled with a multiple porosity representation.A single-phase system using Darcy's Law is not suitable for modeling multiphase flow in liquid-gas systems that is essential for many applications ranging from nuclear waste disposal involving boiling to problems involving a variably saturated zone with oxidationreduction reactions taking place with consequent consumption of oxygen. The EM complex includes diverse hydrologic settings that reflect the variability of geologic and climatic conditions throughout the US. The Hanford, Oak Ridge, and Savannah River sites represent end members of these conditions. The Hanford Site is very dry with a large unsaturated zone, a deep water table, and little atmospheric recharge. The Oak Ridge and Savannah River sites have wet conditions that result in a very shallow water table. The waste forms and contaminant sources vary from simple (increased total dissolved solids) to complex [multiphase gas-liquid and multicomponent air-water Non-Aqueous Phase Liquid (NAPL) at Oak Ridge and Savannah
River] to daunting (mixed NAPL and radioactive waste at Rocky Flats and the Hanford site).

Richards' equation is often used to describe single-phase flow under partially saturated conditions (i.e., the pores are not occupied exclusively by a single phase). As such, it requires the introduction of a relationship between permeability and pressure. Richards' equation is well suited to solve very large numerical problems (millions of degrees of freedom) because it requires only one independent variable per cell. This is accomplished by allowing the gas phase to be a "passive bystander" with zero density and viscosity. There are limitations to this approach. For example, when light or chlorinated hydrocarbons are present, even in very dilute quantities, the accurate representation of Henry's partitioning (which describes the distribution of water between the liquid and gas phases) into the vapor (air) phase can be important. With Richards' equation, the partitioning can be represented, but the subsequent dilution due to air movement cannot. Another problem that can arise in deeper saline aquifers is the change in water density due to changes in brine concentration. In this case, another material balance equation can be introduced and solved in a fully coupled manner (with additional central processing unit and memory requirements) or the density variation can be obtained in a less coupled manner such as explicitly solving the flow and transport independently and calculating the density as a function of concentration. This method, while easier, has numerical stability concerns.

Beyond representing water flow, there are other physical processes that must be included. These include more detailed descriptions of transport, chemical processes, transport of colloids, thermal and mechanical processes, and source terms. In addition, within flow there are many other processes that must be taken into account, the major one being infiltration from various sources including surface water, evaporation, and transpiration.

Transport. Transport is arguably the single most important process that needs to be accurately captured in the environmental management modeling toolset for DOE problems. This is because the rate of transport determines the rate at which contaminants migrate to the biosphere. As formulated for Amanzi, transport refers to the set of physical processes that lead to movement of dissolved and 
solid contaminants in the subsurface, treating the chemical and microbiological reactions that can affect the transport rate through a retardation effect as a separate set of processes. The principal transport processes to be considered are advection, dispersion, and molecular diffusion. In addition, electrochemical migration (8) as related to molecular diffusion are considered.

Biogeochemical Reaction Processes. The reactions that Amanzi will address are those that either add or remove a species from the transport path. In particular, Amanzi will include models for aqueous complexation, or the dissolution and precipitation of materials into or out of solution, sorption of materials onto the surface of other materials, mineral dissolution and precipitation, and microbially mediated reactions. Within the ASCEM framework, the end user will be required to specify which model to use for a given modeling application.

Transport of Colloids. Colloids are very fine particles (such as clay minerals, metal oxides, viruses, bacteria, and organic macromolecules) that range in size between 1 and $10,000 \mathrm{~nm}$ (8) and have high specific surface areas $\left(\sim 300 \mathrm{~m}^{2} / \mathrm{g}\right)$. The generation and mobilization of colloids are considered important issues in contaminant transport, particularly in the transport of radioactive true (intrinsic) colloids (i.e., colloidal $\mathrm{Pu}[\mathrm{IV}]$ and $\mathrm{Pu}[\mathrm{V}]$ ), colloid-assisted transport of radioactive species (i.e., ${ }^{239} \mathrm{Pu},{ }^{237} \mathrm{~Np},{ }^{243} \mathrm{Am}$, and ${ }^{247} \mathrm{Cm}$ ) from high-level radioactive wastes or ${ }^{137} \mathrm{Cs},{ }^{90} \mathrm{Sr}$, and ${ }^{60} \mathrm{Co}$ from low-level radioactive wastes. See (9) for sorption on pseudocolloids (i.e., naturally occurring clay colloids).

The EM complex includes diverse hydrologic settings that involve transport of colloids. Such transport can be a cause of concern when contaminants (such as radionuclides) have sorbed onto these colloids, or the colloids themselves have formed from supersaturation of contaminants. In the case of heavy metals and radionuclides, colloids are responsible for significant transport from the point of release. That is, orders of magnitude larger than what would be expected if solute transport were the main mechanism of contaminant migration. Such transport has been observed at several locations that are part of the DOE complexLos Alamos National Laboratory (LANL), Idaho
National Laboratory (INL), Lawrence Livermore National Laboratory (LLNL), Pacific Northwest National Laboratory (Hanford site) (PNNL), Savannah River National Laboratory (SRNL), and Rocky Flats, Nevada Test Site-which represent a wide spectrum of geological and climatic conditions.

The complexity of colloid-related transport necessitates inclusion of all the known processes. Therefore, it may be necessary to account for different colloid types involved in the transport of a particular species, or for subsets (each representing a range of the particle size distribution) of similar types of colloids. In some of the more complex problems involving transport of a species of interest on multidisperse colloids, it is possible that up to eight equations - two for the main mass components in the gaseous and liquid phases, one for the species of interest (i.e., $\mathrm{PuO}_{2}$ ), and up to five components representing distinct ranges of colloidal sizes - may need to be solved simultaneously. If colloidal and contaminant concentrations are low (i.e., unaffecting the water density), and/or if the flow fields become time invariant within a relatively short time, it is possible that the flow and transport equations can be decoupled and solved separately, speeding up execution. Thus, the code capabilities need to be flexible, accommodating the entire range from very demanding situations necessitating the solution of the fully coupled, strongly nonlinear problem, to more easily solved scenarios in which linearization is possible and flow and transport can be safely decoupled.

The software describing colloid transport will be accessible by all flow-related software developed within the framework of ASCEM. However, it is expected that two- or three-phase flow systems (involving the flow of gaseous, aqueous, and NAPL phases) in geologic media will represent the majority of the problems to be encountered. Additionally, in cases in which the gas phase is not expected to exhibit significant pressure changes, it is expected that a single flow equation (i.e., the Richards' equation, rather than the two full equations for unsaturated water flow) will suffice to describe the hydrologic regime that controls colloid transport. 
Thermal Models. Heat flow and thermal conduction are important aspects of many geochemical systems because heat affects chemical processes through changes in equilibrium and kinetic rate constants. Nonisothermal conditions can also result in buoyancy-driven flow resulting in convection cells and causing fingering phenomena due to differences in density. Incorporation of these processes into the ASCEM model is targeted for development in later years.

Geomechanical and Mechanochemical Models. ASCEM defines geomechanical models broadly as models that describe coupled processes that act to deform or change the mechanical properties of sediment, soil, or rock. Geomechanics can play a very important role in the fluid flow of large groundwater basins. Changes in earth stresses, from either natural (earthquakes) or anthropogenic (pumping), can affect porosity and permeability in aquifers. Similarly, in some EM applications, various processes may cause significant changes in the stress field of an aquifer. For example, remediation treatments may inject large volumes of water at temperatures that are significantly different from the ambient temperature. In these cases, models that include a more formal treatment of mechanics may be required.

In addition to the mathematical/empirical description of geomechanical processes using constitutive equations (i.e., Hooke's Law), ASCEM may also include the underlying mechanistic equations that lead to a specific stress-strain relation. These include coupled mechanical-chemical processes (i.e., swelling, pressure solution, and subcritical crack growth) mediated by transport and stress. It also may include dissolution-precipitation, leading to cementation (strengthening) or reactions leading to weakening (i.e., many silicate to clay weathering reactions). This broad class of geomechanical models is targeted for development in later years.

Source Terms. Cement waste forms and barriers are among the most important of the source terms that need to be considered in a comprehensive Environmental Management simulation tool. Engineered barriers, including cementitious barriers, are used at sites disposing of or contaminated with low-level radioactive waste to enhance performance of the natural environment with respect to controlling the potential spread of contaminants. Drivers for using cementitious barriers include: high radionuclide inventory, radionuclide characteristics (i.e., long half-life and high mobility due to chemical form/speciation), waste matrix properties, shallow water table, and humid climate that provides water for leaching waste.

The ASCEM program will develop several possibilities for dealing with cementitious source terms in the context of the ASCEM modeling and simulation tool:

1. Make use of the simulation tools developed by Cementitious Barriers Partnership (CBP) to represent the cementitious source terms, including the release of contaminants and the collateral effects of cement chemistry (i.e., high $\mathrm{pH}$ ) on groundwater chemistry, or

2. Build on the conceptual models developed by the CBP to develop complementary capabilities using the HPC software tools.

With the first option, the understanding is that the code or codes developed by the CBP will be used as a source term, although possibilities for feedbacks between the processes modeled by the Amanzi software (i.e., flow) and those modeled by the CBP code (i.e., cement degradation) will be limited under this scenario. The CBP will provide a detailed set of process models and their mathematical formulation for later versions of Amanzi.

In addition to the CBP work, a mathematical formulation for glass waste form corrosion over laboratory and geological periods of time is under development as part of the DOE Nuclear Energy Advanced Modeling and Simulation (NEAMS) effort within the DOE. The goal of this effort is to develop one or more rate laws for glass corrosion that can be upscaled from the molecular to microscopic to macroscopic continuum scales. The upscaled rate law will be used in Amanzi.

\section{THE ASCEM DEVELOPMENT LIFE CYCLE}

ASCEM code development will occur in three overlapping phases over the next 4 years, as shown in Figure 3. Each phase continues as capabilities are added based on EM's regulatory needs. ASCEM code development began at the R\&D phase. During 


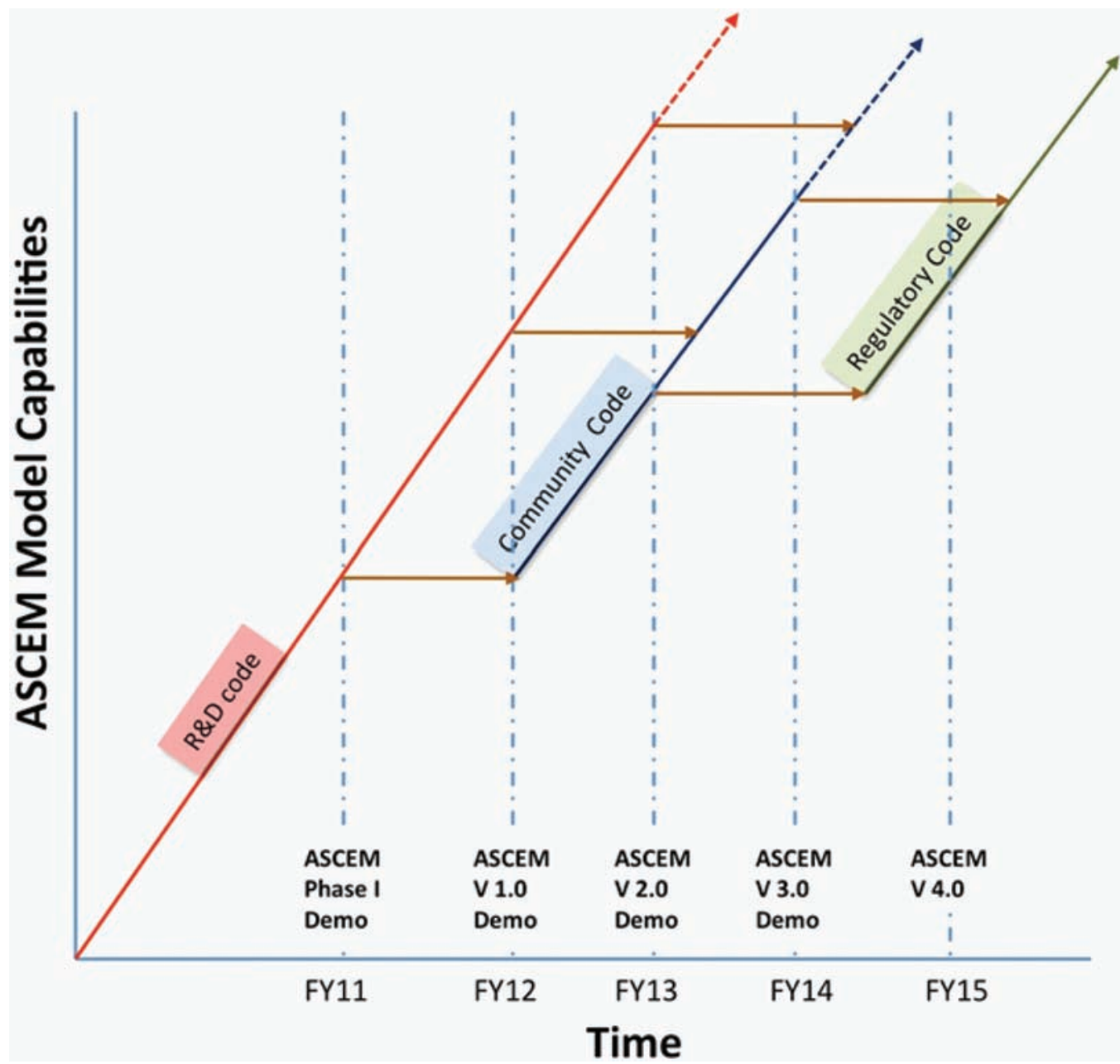

Figure 3. Software life cycle illustration: R\&D code evolves into Community Code (Applied Phase) that is then used as the basis for the Regulatory Code.

this phase, the code requirements and design were defined and developed using standard software quality assurance practices. Once tested and validated, the ASCEM R\&D code will move into the Applied (Community Code) phase for use at the various EM sites for nonregulatory work. The Community Code is the open-source version that can be used by entities (DOE Offices of Fossil Energy, Science, and Nuclear Energy) outside of EM as a shared community model. Finally, at the appropriate times the Community Code will be brought under full Nuclear Quality Assurance Level 1 (NQA-1) quality assurance (QA) requirements and made available to EM for use as their standardized risk and performance assessment code. Thus, at any future time, there may be three ver- sions of the ASCEM code coexisting across the three life cycle phases. Phased code development allows only the full regulatory parts of the ASCEM code to undergo costly NQA-1 requirements, thus allowing development and use of a viable community code without this overhead.

The software QA program as described in the Project Quality Assurance Plan for ASCEM is based on the NQA-1 standard (2004 with 2005 and 2007 Addenda) using a graded approach to the project efforts (based on Subpart 4.2). As the ASCEM code moves from R\&D to applied research and finally to the regulatory (full implementation) phase, the importance and specificity of the QA requirements increase. As the code is developed in each phase, it will be tested and validated 
using appropriate software QA and configuration management practices.

\section{PHASE I DEMONSTRATION (2010)}

The Phase I Demonstration illustrated advances in individual capabilities within the HPC and Platform thrusts. Development of ASCEM capabilities was initiated in 2010 after the software requirements were defined based on EM site modeling needs. The Phase I Demonstration focused on only a subset of developed capabilities. Decisions about which early components of ASCEM to demonstrate were made by considering: 1) feasibility of code/tool advancement within a short time period (primarily September-December 2010), 2) value as a vehicle for communicating ASCEM advances to a variety of stakeholders, and 3) availability and characteristics of available datasets for testing and developing ASCEM components (2).

Salient features of candidate sites and the selection process for the Phase 1 Demonstration were summarized in the "site selection" document (2). The selection process led to a decision by the ASCEM team to focus the ASCEM Phase I Demonstration on the contaminated Savannah River Site (SRS) F-Area around the seepage basin and in particular on the following Platform and HPC components.

1. Platform Data Management, including development of data import, organization, and query tools using a map-based interface with an initial focus on contaminant concentration and hydrostratigraphic variables.

2. Platform Visualization of the hydrostratigraphy, water table, topography, wells, and migration of contaminant plumes through aquifers over time.

3. Platform Uncertainty Quantification, including evaluation of strategies and development of tools to evaluate the sensitivity of model output to parameter suites.

4. HPC capabilities, including the development of: a parallel, unstructured mesh capability; capabilities to perform 3D parallel flow simulation; capabilities to simulate transport of a nonreactive contaminant; and development of a prototype of the Reaction Toolset that includes aqueous speciation, mineral precipitation and dissolution, and sorption.

A major contribution of ASCEM is envisioned to be the integrated nature of the framework. However, the Phase I Demonstration focused on advancing individual components using common datasets from a specific site. Future demonstrations will focus on illustrating integration of expanded capabilities into new versions of ASCEM. The Phase I Demonstration was designed to be tractable and to facilitate the transfer of modeling insights and methods into ASCEM that were developed by NNSA-, EM-, and Basic Energy Research (BER)supported programs.

The Phase I Demonstration work focused primarily on advancing four ASCEM components that are currently under development. Specific Phase I deliverables were developed for each of the four demonstration components with a plan to test ASCEM capabilities using common datasets derived from the contaminated SRS River F-Area Seepage Basins site (1). Use of common datasets from one EM site facilitated coordination of demonstration activities and it encouraged data sharing and integration across ASCEM components under development, and integration across the ASCEM components. This will be the focus of the Phase II Demonstration.

Two supplemental Phase I Demonstration problems focused on Waste Tank Performance Assessment (PA) and Deep Vadose Zone problems. These efforts were identified to do the following: 1) engage a broader set of working groups and end users than represented by the SRS F-Area demonstration; 2) initiate studies focused on linking one or more ASCEM capabilities; 3 ) lay the groundwork for the ASCEM Phase II Demonstration; and 4) to consider additional simulation problems that are distinct from solely subsurface flow and transport but critical to the intended long-term ASCEM development capabilities. The supplementary demonstration activities illustrate advances beyond those obligations identified in the Phase I Demonstration deliverables.

The Phase I Demonstration activities were coordinated by working groups housed under the Site Application Thrust. The working group members provided earth science expertise to the computa- 
tionally oriented ASCEM developers. Working group members helped to define the Phase I Demonstration goals, assembling the necessary input (conceptual models, data process models, and other expert input), providing feedback during the Phase I development phase, and coordinating development of the individual components in the Phase I Demonstration.

Significant progress in advancing all four of the defined ASCEM capabilities was realized during the Phase I Demonstration. The Data Management component adapted and implemented a relational database as well as other open-source, web-based tools to allow end users to easily ingest, browse, filter, graph, query, and output various types of data common to subsurface investigations. Tools were developed to handle both transparent data (such as wellbore concentration and lithology data) and opaque data (such as historical documents). A useful characteristic of the Data Management Toolset is that it can be used iteratively to display and query different subsets of the database based on subregion, characteristic, or parameter range specifications.

As an example of the database management tools, Figure 4 shows a map near the Savannah River F-Area displaying the positions of wells at the site. Adaptations of web-based tools, including Google Maps and the JavaScript plotting package FLOT, were incorporated into ASCEM to enable this functionality, as well as the ability to query the data, and display results in terms of graphs and tables. The right side of Figure 4 shows a list of variables that are available at the wells and clicking a mouse on one of the listings plots the stored data.

The Visualization component of the Phase I Demonstration modified and extended open-source VisIt software to facilitate visualization of data or features common to environmental remediation efforts, such as wellbore geometry, depositional information, hydrostratigraphic surfaces and topography, and the evolution of contaminant plumes. An advantage of these tools is the ability to visualize many different types of data (point, surfaces, volumes) in an uncluttered fashion, the joint visualization of physical features and contaminant concentrations, and the use of slider bars to navigate through temporal datasets (e.g., to view the evolu- tion of a subsurface contaminant plume within a physical framework).

Figure 5 illustrates use of the modified VisIt tool to image the physical framework of the SRS FArea, and Figure 6 shows a different view of the site, including the locations of monitoring wells, Geographic Information System (GIS) data (roads and buildings), surface topography, depositional environment, ${ }^{238} \mathrm{U}$ concentrations in the Lower Aquifer Zone (LAZ) depicted on a white-to-red scale (low to high concentration) at a single point in time, and position of the Gordon Confining Unit (GCU) as a green-colored surface. The figure shows two different perspectives of the plume distribution in 1994. On screen, the viewpoint can be changed by clicking and dragging the mouse.

For the Uncertainty Quantification (UQ) component, ASCEM capabilities were developed to allow a user to choose model parameters and model outputs for a study, and to perform UQ analyses using a variety of different analysis approaches and types. An ASCEM GUI was developed as a framework for the UQ capabilities, which takes advantage of many open-source UQ analysis approaches. A novelty of ASCEM UQ is its integration of the various methods, which are available nowhere else in a single software analysis package. Bringing them together as a single ASCEM UQ tool thus represents a major advance.

Substantial progress was made on development of early prototypes of selected toolsets within the ASCEM Multiprocess HPC Simulator called Amanzi. A parallel, unstructured, hexahedral mesh capability, which can capture complex topography and hydrostratigraphy, was developed (meeting a Phase I deliverable). Building on the unstructured mesh capability, parallel single-phase flow and reactive transport capabilities were developed. Parallel simulations of a period of 10 years were run on 256 processors exceeding the Phase I Demonstration goal of a 2-year simulation run on 100 processors. All of the targeted geochemical processes were implemented in Amanzi's Reaction Toolset (aqueous speciation, mineral precipitation and dissolution, and sorption). A 1D reactive-transport simulation with a five-component model of the SRS F-Area geochemistry was performed and found to be in agreement with two existing codes. A more complex 17-component geochemistry 


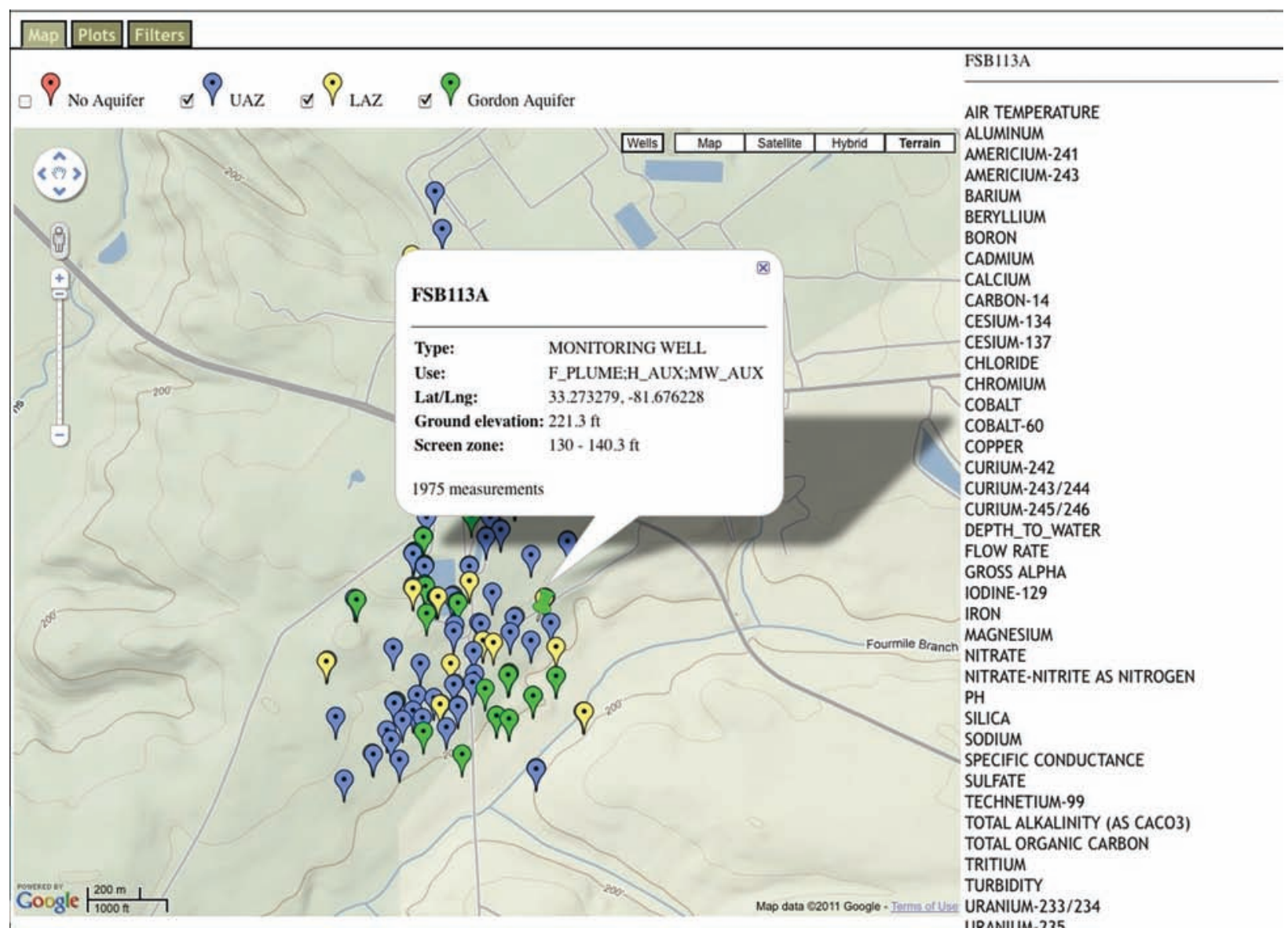

Figure 4. A map-based interface was developed for browsing concentration data using the developed ASCEM map tool. The analytes measured in the selected well are shown on the right. The wells are colored according to the aquifer where the wellbores are screened.

model was run in the full F-Area reactive-transport simulation. Advances were realized with a structured mesh approach as well. A structured Adaptive Mesh Refinement capability that used Amanzi's Reaction Toolset was also explored, exceeding goals established for the Phase I Demonstration. Parallel simulations of the F-Area seepage basins run on 2304 processors demonstrated the potential of this approach to model time-dependent multiphase flows with enhanced fidelity near engineered systems.

ASCEM advances were also realized as part of Waste Tank and Deep Vadose Zone supplemental demonstration problems. The Tank Waste project demonstrated the use of new ASCEM tools to efficiently visualize and interrogate uncertainty results associated with 3D Monte Carlo simulations of potential contamination due to the degradation of closed waste tanks, and an adaptive algorithm was developed using the unstructured approach described above to refine the computational mesh in the vicinity of a discrete tank flow path that controlled by a large contrast in permeability. Conceptual models associated with the Hanford Deep Vadose Zone formed the basis for development of an ASCEM Model Setup Toolset that was linked to the ASCEM UQ GUI. The tool can be used to visualize and link the subsurface layers and their associated properties to the computational grid. The principal advantage of this ASCEM tool is that the multistep process used for translating the conceptual model to the input required for the numerical model can be performed in the same environment, without the need to export data to external software packages.

While the most significant contribution of 


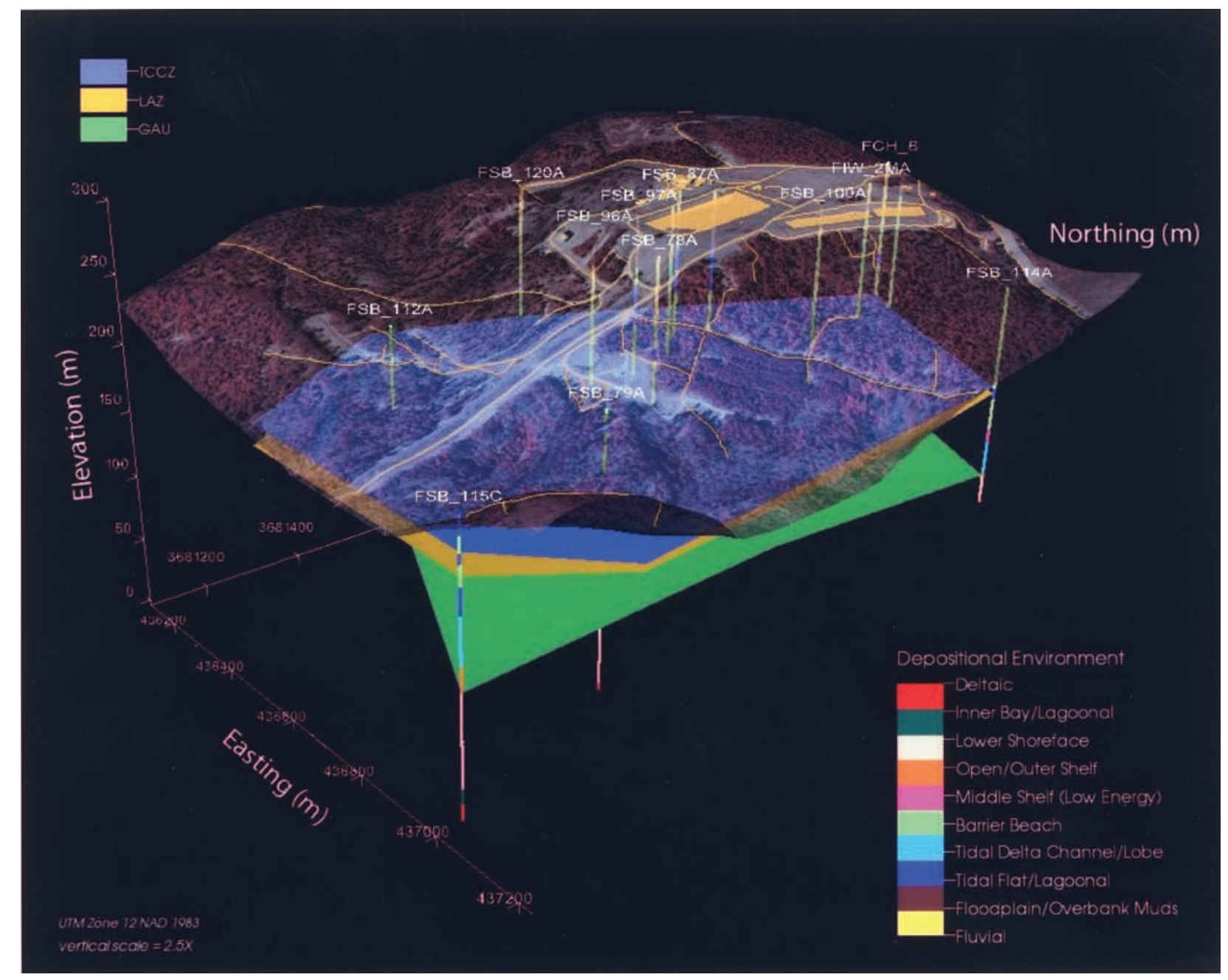

Figure 5. The use of ASCEM capabilities to visualize the physical framework of the SRS F-Area, including the top surfaces of the key stratigraphic layers and depositional environments. The illustration also shows the layout of the monitoring wells, a 3D image of the surface topography along with roads and building footprints, and the distribution of the depositional environments along the well depths.

ASCEM is expected to be its integrated framework and associated computationally efficient, open-source, modular, portable, and accessible characteristics, many of the individual ASCEM components already demonstrate performance or flexibility that exceeds what is available today. The development of an open-source process-based computational framework that can be easily and consistently used across the DOE spectrum of Environmental Management sites is expected to improve cleanup efficacy and decrease overall costs associated with the DOE legacy waste stewardship obligation.

In September of 2010, an initial peer review was conducted on the ASCEM planning process. The review focused on the ASCEM FY10-FY15 Integrated Implementation Plan, which provides the vision and high-level plans for ASCEM for the development phase. The review team noted, "Based on the written information provided to the Independent Peer Review and Assessment (IPRA) Team and particularly as supplemented by discussions with program managers and staff, it appears that the design of ASCEM is well conceived, reasonable and consistent with relevant scientific principles." At a high level the peer review team also noted that, "to ensure that ASCEM proceeds con- 
sistent with DOE needs, compliance with scientific and technical requirements, and other relevant parameters, it is desirable to conduct periodic independent peer reviews." A technical peer review is being planned for the spring of 2011 to supplement the more programmatic review held in September.

\section{CONCLUSIONS}

Based on foundational planning and initial development it can be concluded that the ASCEM program has begun to develop tools and approaches that will provide DOE EM with the resources to support standardized and accurate mod- eling and simulation capability to underpin their risk and performance analysis activities. The full capability with appropriate quality assurance documentation is scheduled to be delivered in 2014 . User releases will be available beginning in 2012 .

ASCEM is a state-of-the-art scientific tool and approach for integrating data and scientific understanding to enable prediction of contaminant fate and transport in natural and engineered systems. The initiative supports the reduction of uncertainties and risks associated with EM's environmental remediation and closure programs by better understanding and quantifying the subsurface flow and contaminant transport behavior in complex geolog-

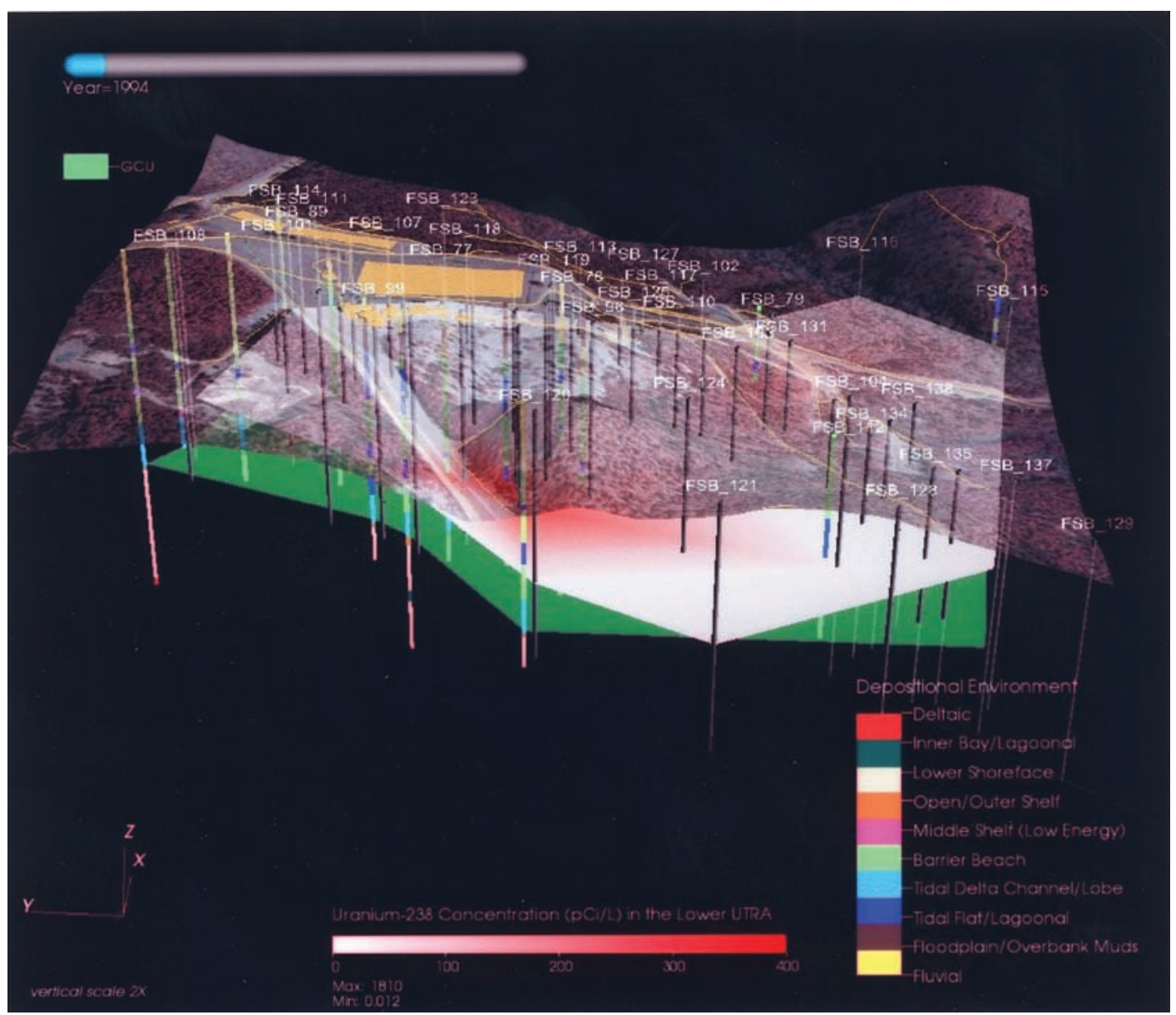

Figure 6. Examples of ASCEM visualization output showing the ${ }^{238} U$ concentration in the LAZ for the year 1994 (the $y$-coordinate is oriented to the North, and the axes scales are the same as in Fig. 5). The illustration also shows the layout of the monitoring wells, a 3D image of the surface topography along with roads and building footprints, the distribution of the depositional environments along the well depths, and the depth of the Gordon Confining Unit (GCU). 
ical systems. This includes the long-term performance of engineered components, including cementitious materials in nuclear waste disposal facilities that may be sources for future contamination of the subsurface.

The ASCEM program focused mainly on planning during the first year. During this time, the team sought input from various stakeholders including site contractors, other DOE offices, and regulators. This input was used in combination with the technical expertise of the team to formulate the ASCEM implementation plan, the requirements documents for each of the thrust areas, and the QA documentation.

Throughout the fourth quarter of calendar year in 2010, the ASCEM team began building the ASCEM computer codes, and developing the Phase I Demonstration. The Phase I Demonstration highlighted several advancements in the areas of the high-performance computing core, data management, uncertainty quantification, and visualization. In 2011, these and other functionalities will be integrated into a cohesive package for release as ASCEM V 1.0.

ACKNOWLEDGMENTS: The authors of this article would like to thank all of the folks working on the ASCEM program who have contributed to the information in this article, including: Deb Agarwal (LBNL), Benjamin Andre (LBNL), Erin Barker (PNNL), John Bell (LBNL), Markus Berndt (LANL), Wes Bethel (LBNL), Gary Black (PNNL), Mike Buksas (LANL), Joann Campbell (LANL), Neil Carlson (LANL), Karl Castleto (PNNL), Jinsong Chen (LBNL), Miles Denham (SRNL), Anthony Drummond (LBNL), Kirsten Fagnan (LBNL), Boris Faybishenko (LBNL), Greg Flach (SRNL), Vicky Freedman (PNNL), Rao Garimell (LANL), Luke Gosink (PNNL), Glenn Hammond (PNNL), David Higdon (LANL), Jennifer Horsman (LBNL), Elizabeth Keating (LANL), Michael Kowalsky (LBNL), Peter Lichtner (LANL), Michael Lijewski (LBNL), Konstantin Lipnikov (LANL), Richard Mills (ORNL), Laura Monroe (LANL), Phil Moore (SRNL), George Pau (LBNL), William Perkins (PNNL), Lori Pritchet (LANL), Sumit Purohit (PNNL), Mark Rockhold (PNNL), Alex Romosan (LBNL), Douglas Sassen (LBNL), Daniel
Schep (SRNL), Karen L. Schuchar (PNNL), Arie Shoshani (LBNL), Dmitryi Silin (LBNL), Mudita Singhai (PNNL), Chandrika Sivarama (PNNL), Nic Spycher (LBNL), Will Stringfe (LBNL), Glenn Taylor (SRNL), Paul Weber (LANL), Yun Wei (LLNL), Mark Williams (PNNL), Signe Wurstner (PNNL), Steve Yabusaki (PNNL), and Yingqi Zhang (LBNL). This article has been authored by the US Department of Energy. The United States Government retains, and by accepting the article for publication, the publisher acknowledges, that the United States Government retains nonexclusive, paid-up, irrevocable, worldwide license to publish or reproduce the published form of this work, or allow others to do so, for United States Government purposes.

\section{ABOUT THE AUTHORS

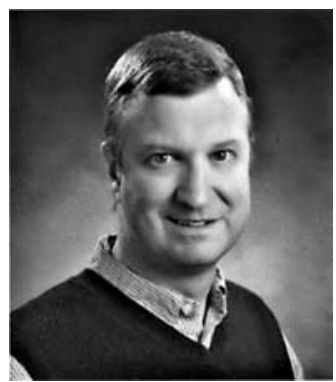

Mark Williamson is the DOE Program Manager for the ASCEM project working through an Intergovernmental Governmental Personnel Act Assignment from Savannah River National Laboratory. Dr. Williamson has spent the last several years working on programs related to Waste Management and the Nuclear Fuel Cycle.

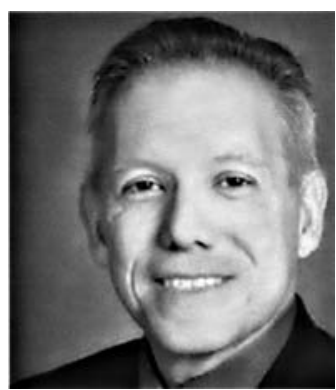

Juan Meza is the Technical Integration Lead for the ASCEM program and head of the High Performance Computing Research Department at Lawrence Berkeley National Laboratory. Dr. Meza oversees work in computational science and mathematics, computer science and future technologies, scientific data management, visualization, numerical algorithms, and application development. 


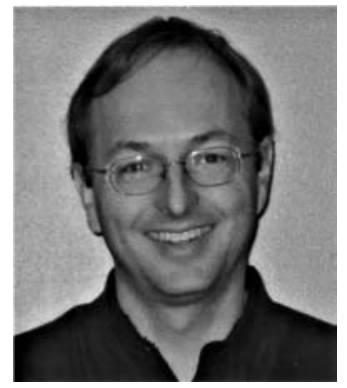

David Moulton is the HPC Thurst Lead for the ASCEM program and is a team leader in the Applied Mathematics and Plasma Physics Group at the Los Alamos National Laboratory. His research focuses on advanced discretizations and multilevel algorithms for simulating flow and reactive transport in heterogeneous porous media.

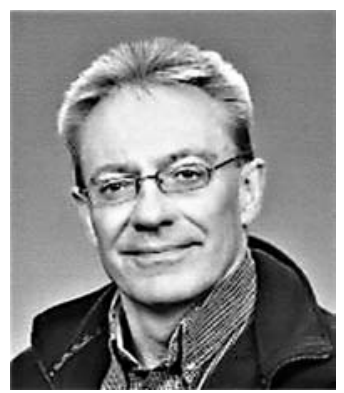

Ian Gorton is the Platform Thrust Lead for the ASCEM program and the Associate Division Director in the Computational Sciences and Mathematics Division at the Pacific Northwest National Laboratory (PNNL). He serves as the Chief Architect for PNNL's Data Intensive Computing Initiative.

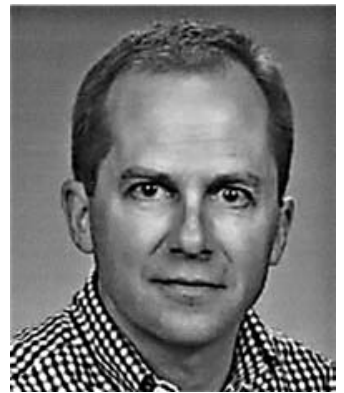

Mark Freshleyis the ASCEM Site Aplications Thurst Lead and manages the Groundwater Remediation and Closure Assessment Projects, which include groundwater monitoring, science and technology, and modeling projects at the Pacific Northwest National Laboratory.

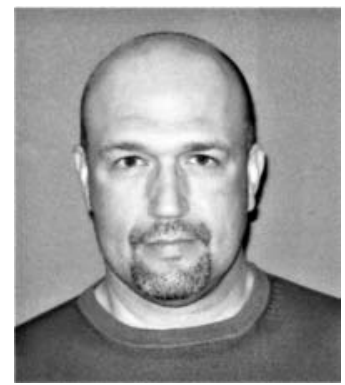

Paul Dixon has performed research and been a technical manager in a variety of energy, nonproliferation, and environmental programs at Los Alamos National Laboratory for the past 21 years. He is the Multi-Laboratory Program Manager of the Advanced Simulation Capability for Environmental Management program for the Department of Energy.

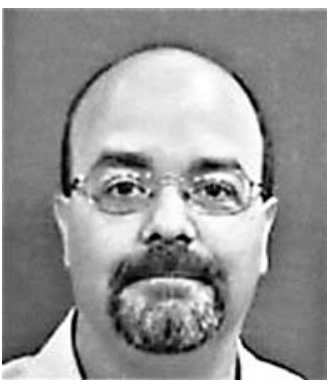

Roger Seitz is the ASCEM User Interface lead and has over 25 years of experience in radioactive and hazardous waste management. He provides technical support to the DOE Office of Environmental Management, the International Atomic Energy Agency (IAEA), and the Savannah River Site on performance assessment (PA) related activities.

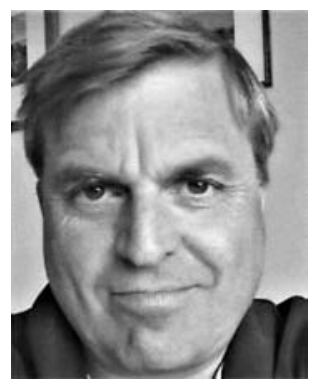

Carl Steefel is the Deputy HPC Thrust lead and a Staff Scientist at Lawrence Berkeley National Laboratory. His current research focuses on reactive transport at multiple scales as it influences such diverse processes as reactive contaminant transport, chemical weathering, water-rock interaction, and biogeochemical cycling. 


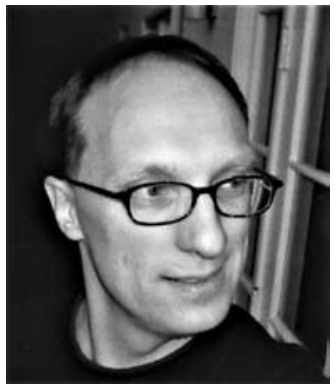

Stefan Finsterle is the Deputy Platform Thurst Lead for ASCEM and has 20 years of experience in the application of multiphase flow and transport simulators to complex subsurface systems. His research focuses on applied geothermal reservoir engineering, nuclear waste isolation, carbon sequestration, and environmental remediation projects.

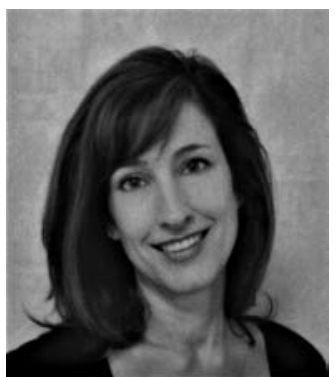

Susan Hubbard is the ASCEM Deputy Site Applications Thrust Lead and is a Senior Scientist at Lawrence Berkeley National Laboratory where she is the Deputy Director of the Earth Science Division and is an Associate Director of the Berkeley Water Center. Her research focuses on advancing the use of geophysical methods for shallow subsurface characterization and monitoring with a particular emphasis on development of data integration methods and application of those methods to environmental problems.]

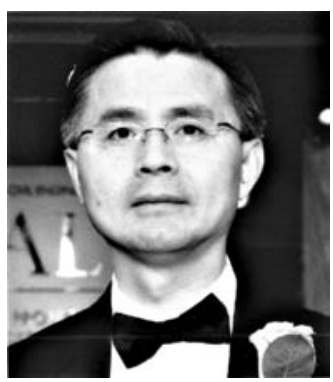

Ming Zhu is Acting Senior Site Program Manager in US Department of Energy's Office of Environmental Management and EM's lead for Interagency Steering Committee for Multimedia Environmental Models. Dr. Zhu served previously as the founding Program Manager for ASCEM.

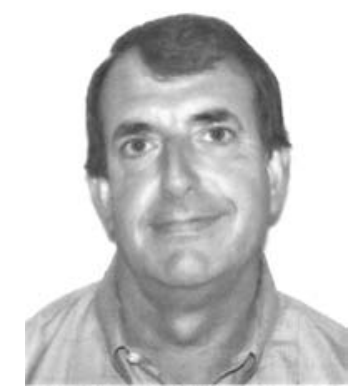

Kurt Gerdes has more than 30 years of experience working in industry, US Navy research facilities, and the Department of Energy. He the Director of the Office of Groundwater \& Soil Remediation within the Office of Environmental Management and manages a program that develops innovative technologies and approaches to address more than over 10,000 areas across the DOE complex requiring groundwater and soil remediation.

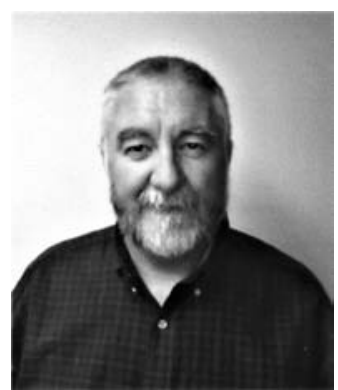

Russ Patterson is Deputy DOE Program Manager for the ASCEM program and is the Program Manager for Performance Assessment and Regulatory Compliance at the US Waste Isolation Pilot Plant. He worked for 14 years in private industry and the US Government in coal exploration, coal mine reclamation, and hazardous waste remediation. Mr. Patterson then spent 7 years at the US Yucca Mountain Nuclear Waste Repository Project (YMP) in charge of scientific site characterization studies.

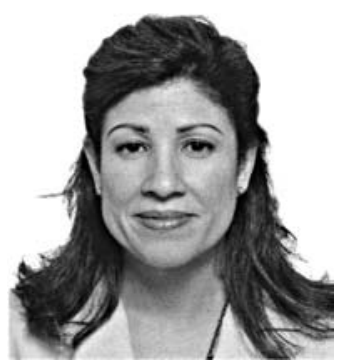

Yvette T. Collazo is the Director for the Office of Technology Innovation \& Development at the US Department of Energy, Office of Environmental Management, in Washington, DC. In this capacity, she is responsible for identifying and advancing technologies that improve the performance of EM's waste processing, groundwater and soil remediation, deactivation and decommissioning, and nuclear materials projects over their life cycles. 


\section{REFERENCES}

1. Advanced Simulation Capability for Environmental Management. Phase I demonstration. ASCEM-SITE-1020100, December 16; 2010.

2. Advanced Simulation Capability for Environmental Management. Site applications thrust site selection task 'Select Phase I Demonstration' milestone 2010. ASCEM-SITE091310-01, October 1; 2010.

3. Cementitious Barriers Partnership. Cooperative research and development agreement for the development of next generation simulation tools to evaluate cementitious barriers and materials used in nuclear applications. Retrieved April 2011, from www.cementbarriers.org.

4. Department Energy, Environmental Management. Engineering and technology Roadmap: Reducing technical uncertainty and risk in the EM program. Retrieved April 2011, from http://www.em.doe.gov/pdfs/FINAL\%20ET\% 20Roadmap\%20_3-5-08_.pdf.

5. Department of Energy. Radioactive waste management. DOE O 435.1, CNG 1; 2007.

6. National Research Council. Advice on the Department of Energy's cleanup technology roadmap: Bridges and gaps. Washington, DC: National Academy Press; 2009.
7. National Research Council. Research needs in subsurface science. Washington, DC: National Academy Press; 2000.

8. Newman, J.; Thomas-Alyea, K. E. Electrochemical systems. Hoboken, NJ: John Wiley and Sons, Inc.; 2004.

9. Office of Civilian Radioactive Waste Management Analysis. Unsaturated zone and saturated zone transport properties (U0100). Technical Report AANL-NBS-HS-000019 REV 00 ICN 02; 2001.

10. Pinder, G. F.; Gray, W. G. Essentials of multiphase flow and transport in porous media. Hoboken, NJ: John Wiley and Sons, Inc.; 2008.

11. US Department of Energy. Journey to excellence: Message from EM Assistant Secretary Triary. Retrieved January 2011, from http://www.em.doe.gov.

12. US Department of Energy, Environmental Management. User suggestions and state of practice for development of ASCEM requirements. Retrieved April 2011, from http:// ascemdoe.org/assets/docs/ ASCEM\%20User\%20Suggestions_Signed3.pdf.

13. US Department of Energy, Environmental Management. Mathematical formulations and requirements for the process models. ASCEM-HPC-10-01, Nov. 10; 2010.

*S. Hubbard's work was conducted as part of the Advanced Simulation Capability for Environmental Management (ASCEM) program, in conjunction with the Office of Environmental Management, of the U.S. Department of Energy under Contract No. DE-AC02-05CH11231. 


\section{DISCLAIMER}

This document was prepared as an account of work sponsored by the United States Government. While this document is believed to contain correct information, neither the United States Government nor any agency thereof, nor The Regents of the University of California, nor any of their employees, makes any warranty, express or implied, or assumes any legal responsibility for the accuracy, completeness, or usefulness of any information, apparatus, product, or process disclosed, or represents that its use would not infringe privately owned rights. Reference herein to any specific commercial product, process, or service by its trade name, trademark, manufacturer, or otherwise, does not necessarily constitute or imply its endorsement, recommendation, or favoring by the United States Government or any agency thereof, or The Regents of the University of California. The views and opinions of authors expressed herein do not necessarily state or reflect those of the United States Government or any agency thereof or The Regents of the University of California.

Ernest Orlando Lawrence Berkeley National Laboratory is an equal opportunity employer. 